Copyright (C) 2014 IEEE. Personal use of this material is permitted. Permission from IEEE must be obtained for all other uses, in any current or future media, including reprinting/republishing this material for advertising or promotional purposes, creating new collective works, for resale or redistribution to servers or lists, or reuse of any copyrighted component of this work in other works. 


\section{Antenna Rotation Error Tolerance for a Low-Frequency Aperture Array Polarimeter}

\author{
Adrian T. Sutinjo and Peter J. Hall
}

\begin{abstract}
We present antenna rotation error tolerance analysis for a polarimeter consisting of dual-linearly polarized dipole-like elements. Treating the elements as a phased array and expressing the measurement basis as circularly polarized (CP) results in a concise expression for the Jones matrix for the array. For the type of elements being considered, the matrix shows that the intrinsic cross-polarization ratio (IXR) of the array at the intended beam scanning direction is unaffected by small rotation errors. For random rotation error and very large number of elements, we further find that the relative Jones matrix estimation error converges to that of the error-free case at the intended beam scanning direction; however, the effect of element rotation error on array directivity and radiation pattern remains. Recasting the analysis with the array observing an unpolarized source, a relation between rotation error and cross-polarization "leakage" is obtained, wherein similar trends with very large number of elements hold true. Practical examples involving "large" number of elements such as the low frequency Square Kilometre Array are discussed.
\end{abstract}

Index Terms- Antenna theory, Radio astronomy, Polarimetry

\section{INTRODUCTION}

Precise alignment of dipole-like antennas for low-frequency aperture arrays (LFAAs) is generally thought to be of great importance especially for polarimetry, where rotation misalignment translates to raw cross-polarization error. In recent low-frequency telescopes, this has been accomplished via elemental alignment to pre-surveyed metallic grids. One example of this is the Murchison Widefield Array (MWA) "tile" [1], [2] (a regularly spaced array of 16 "bowties" combined through an analog beamformer, as seen in Fig. 1) where the elements are clipped to the mesh wires that have been pre-surveyed to align with N-S and E-W. A similar grid assisted method was also employed in LOFAR [3], [4] ${ }^{1}$.

Although this method was satisfactory in the MWA and might scale to the Square Kilometre Array (SKA) LFAA, we are motivated here to revisit rotational error tolerance for the LFAA for a few of reasons. Firstly, as a result of bandwidth requirement for the SKA LFAA (tentatively, 70-450 $\mathrm{MHz}$ [6] or $50-300 \mathrm{MHz}$ [7]), recently developed candidate elements have been designed to be wideband and directional in absence of a metallic ground plane. A couple of cases in point are the zenith-directed, and nearly azimuthally pattern-symmetric, conical spiral antenna [8] and the logperiodic antenna (SKALA) [9], [10]. Hence, the possibility of having to align the antennas in the absence of a grid and the tolerance involved should be considered as well as the longer

The authors are with International Centre for Radio Astronomy Research/Curtin Institute of Radio Astronomy (ICRAR/CIRA), Curtin University, Bentley, WA 6102, Australia (e-mail: adrian.sutinjo@gmail.com).

${ }^{1}$ Another alignment method using a spotting scope to a distant geographical marker was employed in the long wavelength array (LWA) [5]. Due to the lack of similarly distant landmark at the Murchison Radio-Astronomy Observatory, this method is not being considered. term consideration of deploying very high number of elements and/or "stations" (e.g., the SKA1 LFAA Baseline Design calls for 289 elements per station and 911 stations [7]. A "station" is a beamformed array similar to an MWA "tile" but consisting of more elements.). Secondly, in deploying a prototype array at the Murchison Radio-Astronomy Observatory (MRO) [10], [11] using a compass for alignment, we found a standard deviation of approximately $5^{\circ}$; a review is needed to determine if this is acceptable. Finally, a figure-of-merit for radio polarimeters, the intrinsic cross-polarization ratio (IXR), which is more fundamental than raw cross-polarization ratios has recently been introduced $[12]^{2}$. A review of rotation error tolerance in terms of IXR may reveal a more fundamental view of error tolerance than suggested by raw cross-polarization.

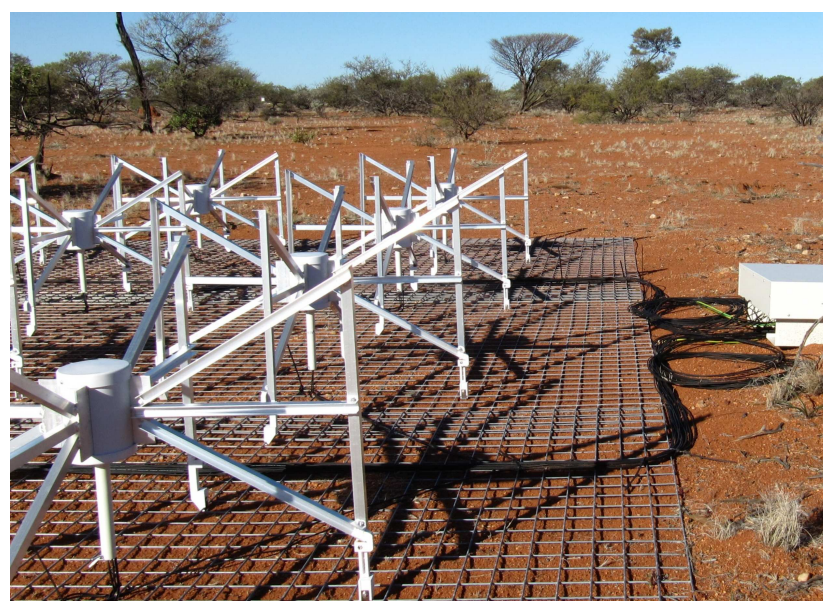

Figure 1. A close-up photo of an MWA tile placed on a $5 \mathrm{~m} \mathrm{X} 5 \mathrm{~m}$ wire mesh. The elements are connected to an analog beamformer seen on the right.

The aim of the analysis here is to estimate the degree of alignment required in the deployment of large number of elements. This is intended to provide input to next generation MWA and other low frequency arrays such as the SKA LFAA [7]. The division in hierarchy between phased array beamforming and correlation for LFAA remains a topic of discussion [14]. However, in our scope, we make the assumption that a (large) number elements are beamformed as a simple phased array to form a "station" beam. A summary of this work highlighting key results is presented in [15]; here, more detailed steps on the derivations and discussions on large and very large number of elements are given.

The rest of the paper is organized as follows. Sec. II details the Jones matrix derivation for the phased array of dipolelike elements including rotation error. In sections III and IV, the impact of the error is quantified in terms of fundamental

${ }^{2}$ Jones IXR is a fundamental figure-of-merit in that it is related to the condition number of the Jones matrix and is independent of coordinate systems. It provides an upper bound estimate for total relative error of the sky Jones vector [12], [13]

$$
\frac{\|\Delta \mathbf{e}\|}{\|\mathbf{e}\|} \lesssim\left(1+\frac{2}{\sqrt{\mathrm{IXR}}}+\ldots\right)\left(\frac{\|\Delta \mathbf{J}\|}{\|\mathbf{J}\|}+\frac{\|\Delta \mathbf{f}\|}{\|\mathbf{f}\|}\right)
$$

where $\|\Delta \mathbf{J}\| /\|\mathbf{J}\|$ and $\|\Delta \mathbf{f}\| /\|\mathbf{f}\|$ are the relative errors for the Jones matrix estimation and the measured vector, respectively. 
figure-of-merit and Jones polarimetry input errors. Additional calculations involving a polarimeter illuminated with a single unpolarized source are presented in Sec. V. Finally, the implications of the findings are summarized in Sec. VI.

\section{JONES MATRIX DERIVATION}

Using linear polarization (LP) bases, the polarimeter response for a dual-polarized orthogonal Hertzian-dipole pair with rotation error (superscript ${ }^{i}$ refers to pair $i$ as shown in Fig. 2 for an array of $\mathrm{N}$ elements) may be written as:

$$
\mathbf{f}^{i}=\mathbf{E}^{i} \mathbf{J}_{L} \mathbf{e}
$$

where $\mathbf{f}^{i}=\left(f_{x}^{i}, f_{y}^{i}\right)^{T}$ indicates the measured vector, $\mathbf{e}=$ $\left(e_{\theta}, e_{\phi}\right)^{T}$ denotes the sky Jones vector, and

$$
\mathbf{J}_{L}=\left(\begin{array}{cc}
J_{x \theta} & J_{x \phi} \\
J_{y \theta} & J_{y \phi}
\end{array}\right)
$$

is the Jones matrix for the pair (assumed identical for all pairs in the array). We point out that $\mathbf{f}^{i}, \mathbf{J}_{L}$, and $\mathbf{e}$ in (2) are functions of $\phi$ and $\theta$ (spherical coordinate). The rotation error matrix in (2) for pair $i$ is given by

$$
\mathbf{E}^{i}=\left(\begin{array}{cc}
\cos \delta_{i} & \sin \delta_{i} \\
-\sin \delta_{i} & \cos \delta_{i}
\end{array}\right)
$$

For orthogonal Hertzian dipoles, $(2,4)$ are exact for any $\delta_{i}$ and $(\phi, \theta)$ as the IXR pattern is invariant with respect to $\phi$. However for practical (such as half-wavelength) dipoles, some azimuthal IXR undulations exist. In these cases, $(2,4)$ are not exact but they provide very good approximation for a wide range of $\theta$ if $\delta_{i}$ is restricted to small angles (see Appendix A for a quantitative example) such that the equality sign in (2) can be replaced by an approximate $\operatorname{sign}(\approx)$. Note that $(2$, 4) are not applicable to antennas with large azimuthal IXR variations such as a dual-polarized Vivaldi elements [16 ${ }^{3}$.
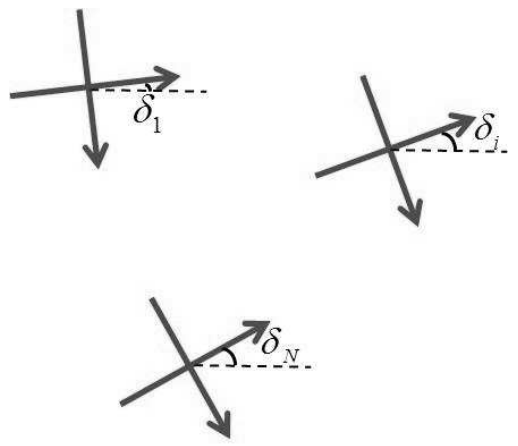

Figure 2. Rotational misalignment in an array of identical cross-dipole elements (top view). $\delta_{i}$ is the rotation error of pair $i$.

\footnotetext{
${ }^{3}$ This restriction is not a concern within our scope as many low-frequency aperture arrays such as the MWA and LOFAR do use dipole-like elements (where only small undulations are expected; case in point: MWA bowties [17]). Furthermore, the practice of using dipole-like elements is likely to continue in the future as reflected in the SKA LFAA Baseline Design [7]. Regarding the presence of ground plane below the dipoles (such as in MWA or LOFAR), it should be pointed out that the treatment here is fully applicable. It may be accounted for in the Jones matrix (3) by a scalar array factor multiplier which does not affect the results.
}

Assuming that the antenna pairs are identical ${ }^{4}$, the polarimeter response for a planar array is given by

$$
\mathbf{f}=\frac{1}{N}\left(\sum_{i=1}^{N} e^{j\left(k_{x} x_{i}+k_{y} y_{i}+\varphi_{i}\right)} \mathbf{E}^{i}\right) \mathbf{J}_{L} \mathbf{e}
$$

where $k_{x}=(2 \pi / \lambda) \sin \theta \cos \phi, k_{y}=(2 \pi / \lambda) \sin \theta \sin \phi, x_{i}, y_{i}$ are the element positions, and $\varphi_{i}$ is the phase shift for pair $i$. Converting the array measurement basis from LP to $\mathrm{CP}^{5}$

$$
\mathbf{f}_{C}=\frac{1}{N} \mathbf{R}\left(\sum_{i=1}^{N} e^{j\left(k_{x} x_{i}+k_{y} y_{i}+\varphi_{i}\right)} \mathbf{E}^{i}\right) \mathbf{J}_{L} \mathbf{e}
$$

where $\mathbf{f}_{C}=\left(f_{l}, f_{r}\right)^{T}$ (subscripts ${ }_{l, r}$ refer to left hand (LH) and right hand $(\mathrm{RH}) \mathrm{CP}$, respectively) and the transformation matrix is

$$
\mathbf{R}=\frac{1}{\sqrt{2}}\left(\begin{array}{cc}
-j & 1 \\
1 & -j
\end{array}\right)
$$

Note that $\mathbf{R}$ may be taken into the summation in (6). Recognizing

$$
\mathbf{R E}^{i}=\left(\begin{array}{cc}
e^{-j \delta_{i}} & 0 \\
0 & e^{j \delta_{i}}
\end{array}\right) \mathbf{R}
$$

we note that rotation error manifests as oppositely signed phase shifts for the LH and RH components, respectively [18].

The array factors due to the positive and negative phase errors are given by

$$
F^{ \pm}=\frac{1}{N} \mathbf{s}^{T} \mathbf{d}^{ \pm}
$$

where $\mathbf{s}=\left[e^{j\left(k_{x} x_{1}+k_{y} y_{1}+\varphi_{1}\right)} \ldots e^{j\left(k_{x} x_{N}+k_{y} y_{N}+\varphi_{N}\right)}\right]^{T}$ and $\mathbf{d}^{+}=\left(\mathbf{d}^{-}\right)^{*}=\left[e^{j \delta_{1}} \cdots e^{j \delta_{N}}\right]^{T}$. As a result, we may now write (6) more concisely

$$
\begin{aligned}
\mathbf{f}_{C} & =\left(\begin{array}{cc}
F^{-} & 0 \\
0 & F^{+}
\end{array}\right) \mathbf{R J}_{L} \mathbf{e} \\
& =\mathbf{F R J}_{L} \mathbf{e}
\end{aligned}
$$

The rotation error for the array is now expressed as diagonal matrix that pre-multiplies the error-free Jones matrix. For ease of interpretation, (10) is expressed using CP sky basis

$$
\mathbf{f}_{C}=\mathbf{F} \mathbf{J}_{C} \mathbf{e}_{C}
$$

where $\mathbf{J}_{C}=\mathbf{R} \mathbf{J}_{L} \mathbf{R}^{H}$ is the CP Jones matrix and $\mathbf{e}_{C}=$ $\left(e_{l}, e_{r}\right)^{T}$ is the CP sky vector.

\footnotetext{
${ }^{4}$ We should point out that (5) applies when mutual coupling is negligible, such as for an irregularly spaced sparse array. However, in the presence of mutual coupling, if the embedded element Jones matrices may be assumed identical and rotation error is small, (5) may be used

${ }^{5}$ This transformation is performed for convenience as it leads to an intuitive interpretation of the results. Note that multiplication by unitary matrix $\mathbf{R}$ only amounts to a change of coordinate system and does not alter the IXR [12], [17].
} 


\section{EFFECTS OF ROTATION ERROR ON IXR}

In this section, we examine if rotation error fundamentally alters the polarimeter's figure-of-merit. The Jones intrinsic cross-polarization ratio is given by [12]

$$
\operatorname{IXR}=\left(\frac{\sigma_{\max } / \sigma_{\min }+1}{\sigma_{\max } / \sigma_{\min }-1}\right)^{2}
$$

where $\sigma_{\max }$ and $\sigma_{\min }$ refer to the maximum and minimum singular values of the Jones matrix in question which in this case is $\mathbf{J}_{C}^{e r r}=\mathbf{F} \mathbf{J}_{C}$. Numerical computation of IXR is straightforward for a given array with element rotation error. However, it is useful to understand rotation effect on IXR analytically; hence, we discuss the following special case.

At the intended beam scanning direction $\left(\theta_{t}, \phi_{t}\right)$, $k_{x}\left(\theta_{t}, \phi_{t}\right) x_{i}+k_{y}\left(\theta_{t}, \phi_{t}\right) y_{i}+\varphi_{i}=0$. In this case,

$$
F_{t}^{ \pm}=\frac{1}{N} \sum_{i=1}^{N} e^{ \pm j \delta_{i}}
$$

where the subscript $t$ has been introduced as a shorthand for $\left(\theta_{t}, \phi_{t}\right)$. Note that

$$
F_{t}^{+}=\left(F_{t}^{-}\right)^{*}
$$

such that

$$
\begin{aligned}
\mathbf{f}_{C t} & =F_{t}^{-}\left(\begin{array}{cc}
1 & 0 \\
0 & 1 \angle\left(-2 F_{t}^{-}\right)
\end{array}\right) \mathbf{J}_{C} \mathbf{e}_{C} \\
& =F_{t}^{-} \mathbf{M}^{\text {err }} \mathbf{J}_{C} \mathbf{e}_{C}
\end{aligned}
$$

Note that $\mathbf{M}^{e r r}$ in (15) is a unitary matrix. As IXR is unitarily invariant [12], [17] and is unaffected by scalar multiplication, we find

$$
\operatorname{IXR}\left(\mathbf{J}_{C}^{e r r}\left(\theta_{t}, \phi_{t}\right)\right)=\operatorname{IXR}\left(\mathbf{J}_{C}\left(\theta_{t}, \phi_{t}\right)\right)
$$

In other words, if the rotation error model described by $(2,4)$ is exact (e.g., for dual-polarized Hertzian dipoles) the fundamental figure-of-merit for a polarimeter at the intended beam scanning direction (for a discussion regarding IXR at $(\theta, \phi)$, see Appendix B). suffers no degradation in comparison to the error-free case. Again, in practical cases where the rotation error model is an approximation for small errors, the equality sign in $(16)$ is to be replaced with an approximate $(\approx)$ sign. Note that our treatment so far has been deterministic such that (16) applies to random and non-random error distributions for any $N$. In the case of random errors, our finding is consistent with recent numerical simulation result from a 16-element SKALA array with $\pm 15^{\circ}$ random error where negligible effect on IXR was reported [10].

In view of (16) and (1), if the total relative input error to the polarimeter is held constant, the relative output error upper bound remains unchanged. Naturally, this leads to the question of how much the total relative input error degrades in the presence of rotation error. This is discussed in next.

\section{JONES POLARIMETRY INPUT ERRORS FOR LARGE $N$}

The previous section deal with the first factor in (1). This section addresses the effect of rotation error on the second factor in (1) for large and very large $N$.

\section{A. Input Errors for $N \rightarrow \infty$}

In remainder of this document, we assume $\delta_{i}$ to be a Gaussian random variable with zero mean ${ }^{6}$ and variance $\sigma^{2}$ (independent and identically distributed for $i=1 \ldots N$ ) and restrict the treatment to $\left(\phi_{t}, \theta_{t}\right)$. The mean of $F_{t}$ is

$$
\mathrm{E}\left[F_{t}\right]=e^{-\sigma^{2} / 2}
$$

From array tolerance theory [20]-[23], directivity relative to the error-free case is given by

$$
\frac{D}{D_{0}}=\frac{1}{1+\sigma^{2}} \approx \mathrm{E}\left[F_{t}\right]^{2}
$$

which is plotted in Fig. 3.

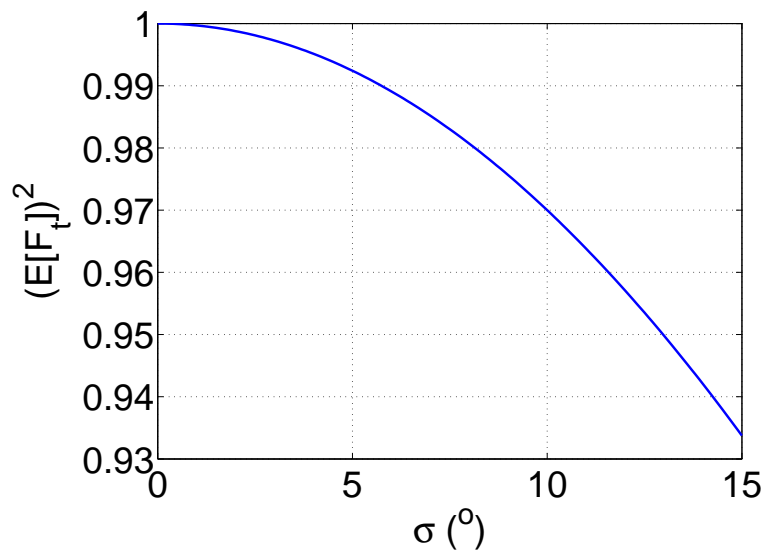

Figure 3. $D / D_{0}$ vs. rotation error. $\sigma=5.7^{\circ}, 10^{\circ}, 13^{\circ}$ result in directivity degradations of $1 \%, 3 \%, 5 \%$, respectively

Variances for the real and imaginary parts of $F_{t}$ for large $N$ are given by [24]

$$
\begin{aligned}
\sigma_{r}^{2} & =\frac{1+e^{-2 \sigma^{2}}}{2 N}-\frac{e^{-\sigma^{2}}}{N} \\
\sigma_{i}^{2} & =\frac{1-e^{-2 \sigma^{2}}}{2 N}
\end{aligned}
$$

These standard deviations are plotted in Fig. 4. Note that $\sigma_{i}$ is dominant. Also, for $\sigma_{i}<<1, \sigma_{i}^{2} \approx \sigma^{2} / N$.

1) $\|\Delta \mathbf{J}\| /\|\mathbf{J}\|$ Estimate: In this asymptotic case, variances for the real and imaginary parts of $F_{t}(\propto 1 / N)$ vanish, and we obtain

$$
\mathbf{J}_{C}^{e r r} \approx \mathrm{E}\left[F_{t}\right] \mathbf{J}_{C}
$$

As $\mathrm{E}\left[F_{t}\right]$ is a scalar factor, it is reasonable to expect that it may be removed by appropriate normalization such that $\mathbf{J}_{C}^{\text {err }} \approx \mathbf{J}_{C}$. This implies that, for large $N,\|\Delta \mathbf{J}\| /\|\mathbf{J}\|$ may be assumed to be the same as the rotation error-free case.

\footnotetext{
${ }^{6}$ Note that "zero" mean introduces no additional constraint to the analysis. Non-zero mean error can be "zeroed" by re-adjusting the reference for rotation error in Fig. 2 to that non-zero mean. We further point out that the lack of a priori knowledge regarding this non-zero mean presents no complication. If this array is a constituent of a multiple baseline interferometer [19], in the CP basis and given our foregoing assumptions, non-zero mean errors can be represented as a non-direction dependent diagonal matrix $([\exp (-j \delta), 0 ; 0, \exp (j \delta)]$, where $\delta$ is that non-zero mean error) which multiplies $\mathbf{F}$ in (10). The $\delta$ terms are subsumed as complex gain phase and are solved during the "complex gain" calibration step.
} 


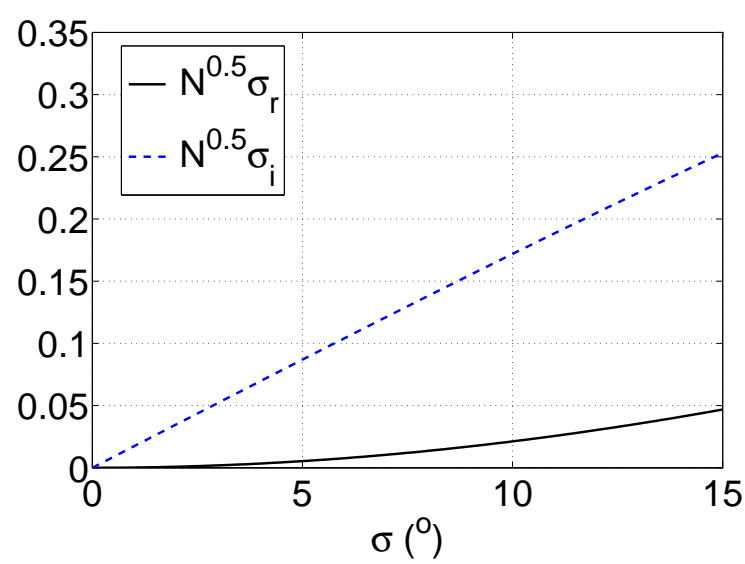

Figure 4. Standard deviations $\times \sqrt{N}$ of the real and imaginary components of $F_{t}$ vs. rotation error.

2) $\|\Delta \mathbf{f}\| /\|\mathbf{f}\|$ Estimate: Rigorous analysis of measurement error requires a noise model, which is beyond our scope. However, it suffices here to assume that the array sensitivity at a given frequency is proportional to the array directivity

$$
\frac{A_{e}}{T_{\text {sys }}} \propto D
$$

(i.e., the system temperature, $T_{\text {sys }}$, is assumed constant with small changes in directivity). Therefore, we assume directivity degradation as a function of rotation error to be proportional to the (square of) relative error in the measurement. Fig. 3 demonstrates that directivity degrades with increasing rotation error, which for large $N$ is the dominant source of degradation. It is reasonable here to set rotation error budget commensurate to a level of acceptable directivity degradation. For instance, directivity degradation of $5 \%$ or less requires $\sigma \leq 13^{\circ}$. Note that rotation error also degrades radiation pattern properties such as sidelobe levels which may be accounted for as required [20]-[23].

\section{B. Input Errors for Large $N$}

Similar arguments as found in Sec. IV-A may be made for large but finite $N$. The question is what is a large $N$ ? $N$ may be considered sufficiently large if $\sigma_{i}<<E\left[F_{t}\right] \approx 1$. Referring to $N^{0.5} \sigma_{i}$ curve in Fig. 4 and taking $\sigma=10^{\circ}$ as an example, $N \approx 17^{2}=289$ results in $\sigma_{i} \approx 0.01$. Similarly, for $\sigma=5^{\circ}$, $N \approx 9^{2}=81$ is sufficiently large.

\section{UNPOLARIZED SOURCE}

The previous sections made no assumption about the polarization property of the source. However, as radio astronomy sources are generally unpolarized or are weakly polarized [25], we now refine our calculations to account for this.

Carrying on the analysis from the previous section and assuming that there exists a single point source at the intended beam direction $\left(\theta_{t}, \phi_{t}\right)$, the autocorrelation matrix may be written as

$$
\mathbf{V}=\mathbf{J B J}^{H}
$$

where $\mathbf{B}=\left\langle\mathbf{e} \mathbf{e}^{H}\right\rangle$ is the brightness matrix and $\mathbf{J}$ is the Jones matrix for the entire array at the intended beam direction. Let (22) represent the rotation error-free case. Introducing rotation error to all $N$ elements in the array while keeping the same source at $\left(\theta_{t}, \phi_{t}\right)$, we obtain

$$
\tilde{\mathbf{V}}=\tilde{\mathbf{J}} \mathbf{B} \tilde{\mathbf{J}}^{H}
$$

where : indicates the presence of error. To extract the brightness matrix from (23) without accounting for rotation errors (assuming no error-correction scheme is involved), we write

$$
\begin{aligned}
\tilde{\mathbf{B}} & =\mathbf{J}^{-1} \tilde{\mathbf{V}}\left(\mathbf{J}^{H}\right)^{-1} \\
& =\mathbf{J}^{-1} \tilde{\mathbf{J}} \mathbf{B} \tilde{\mathbf{J}}^{H}\left(\mathbf{J}^{H}\right)^{-1}
\end{aligned}
$$

For an unpolarized source, $\mathbf{B}=\mathbf{I} / 2$ [26]. For this special case, (24) simplifies to

$$
\tilde{\mathbf{B}}=\frac{1}{2} \mathbf{J}^{-1} \tilde{\mathbf{J}} \tilde{\mathbf{J}}^{H}\left(\mathbf{J}^{H}\right)^{-1}
$$

Assuming $\mathrm{CP}$ bases $^{7}$ and small rotation errors as before

$$
\tilde{\mathbf{B}} \approx \frac{1}{2} \mathbf{J}^{-1} \mathbf{F} \mathbf{J J}{ }^{H} \mathbf{F}^{H}\left(\mathbf{J}^{H}\right)^{-1}
$$

where $\mathbf{F}=F_{t}^{-} \mathbf{M}^{e r r}$ as per (15). Note that the interjection of the diagonal matrix $\mathbf{F}$ and introduces off-diagonal "leakage" (i.e., non-zero cross-polarization correlation: $\left.\left\langle e_{l} e_{r}^{*}\right\rangle,\left\langle e_{l} e_{r}^{*}\right\rangle\right)$ terms in $\tilde{\mathbf{B}}$. Multiplying the matrices, it can be shown that

$$
\begin{aligned}
& \tilde{B}_{1,2}=\tilde{B}_{2,1}^{*}= \\
& \left(J_{l l} J_{r l}\right)^{*}\left(F_{t}^{-}-F_{t}^{+}\right)\left(F_{t}^{-} J_{l l} J_{r r}-F_{t}^{+} J_{l r} J_{r l}\right)+ \\
& J_{l r} J_{r r}\left(F_{t}^{-}-F_{t}^{+}\right)\left(-F_{t}^{-} J_{l r} J_{r l}+F_{t}^{+} J_{r r} J_{l l}\right)^{*}
\end{aligned}
$$

where the scalar multiplier $1 /\left(2|\operatorname{det} \mathbf{J}|^{2}\right)$ has been suppressed. Estimates may be obtained by assuming $J_{l l} \approx J_{r r} \approx 1, F_{t}^{ \pm} \approx$ 1 , and $J_{l r} \approx J_{r l}<<1$, such that the leakage levels may be approximated as

$$
\tilde{B}_{1,2} \approx 4 j J_{\Delta} \operatorname{Im}\left(F_{t}^{-}\right)
$$

where $J_{\Delta}$ has been introduced to refer to $J_{r l}$ or $J_{l r}$.

As in Sec. IV, let $\delta_{1 . . N}$ be independent Gaussian random variables with zero mean and variance $\sigma^{2}$. Referring to (19), the variance of the leakage levels for small $\sigma$ is

$$
\operatorname{VAR}\left[4 J_{\Delta} \operatorname{Im}\left(F_{t}^{-}\right)\right] \approx \frac{\left(4 J_{\Delta} \sigma\right)^{2}}{N}
$$

We note again that the variance vanishes with very large number of elements. For finite number of elements and leakage with a certain desired standard deviation, one may calculate the minimum number of elements required for a given $\sigma$ and $J_{\Delta}$. This is illustrated in Tab. I for $0.5 \%$ leakage.

Further calculations based on an SKA LFAA station are reported in Tab. II. Here the number of elements and leakage standard deviation are fixed at $N=289$ [7] and $0.5 \%$, respectively. Note that a moderate $J_{\Delta}$ of 0.2 requires rotation standard deviation of $\approx 6.1^{\circ}$ whereas a "good" $J_{\Delta}$ of 0.1 can tolerate rotation error of $\approx 12^{\circ}$.

$$
\begin{aligned}
& { }^{7} \text { such that the Jones matrix is given by } \\
& \qquad \mathbf{J}=\frac{1}{2}\left(\begin{array}{cc}
J_{l l} & J_{l r} \\
J_{r l} & J_{r r}
\end{array}\right)
\end{aligned}
$$

where the subscripts $l$ and $r$ refer to left and right hand circular polarizations, respectively. 
Table I

NUMBER OF ELEMENTS REQUIRED TO ACHIEVE LEAKAGE WITH STANDARD DEVIATION OF $0.5 \% .1-\left(\mathrm{E}\left[F_{t}\right]\right)^{2}$ IS THE DIRECTIVITY DEGRADATION IN PERCENT. $J_{\Delta}$ AND $\sigma_{i}$ ARE VOLTAGE QUANTITIES.

\begin{tabular}{ccccc}
$J_{\Delta}$ & $\sigma\left({ }^{o}\right)$ & $N$ & $1-\left(\mathrm{E}\left[F_{t}\right]\right)^{2}(\%)$ & $\sigma_{i}$ \\
\hline 0.1 & 5 & 49 & 0.8 & 0.0125 \\
\hline 0.1 & 10 & 195 & 3 & 0.0125 \\
\hline \hline 0.33 & 5 & 531 & 0.8 & 0.004 \\
\hline 0.33 & 10 & 2123 & 3 & 0.004 \\
\hline
\end{tabular}

Table II

ROTATION ERROR STANDARD DEVIATION $(\sigma)$ REQUIRED TO ACHIEVE LEAKAGE WITH STANDARD DEVIATION OF $0.5 \%$ FOR $N=289$.

\begin{tabular}{cccc}
$J_{\Delta}$ & $\sigma\left(^{o}\right)$ & $1-\left(\mathrm{E}\left[F_{t}\right]\right)^{2}(\%)$ & $\sigma_{i}$ \\
\hline 0.1 & 12.2 & 4.4 & 0.012 \\
\hline 0.2 & 6.1 & 1.1 & 0.006 \\
\hline 0.33 & 3.7 & 0.4 & 0.004 \\
\hline
\end{tabular}

It is interesting to note that, in contrast to previous section where no source polarization was defined, the definition of the unpolarized source provides information on the interplay between rotation error and cross-polarization levels of the element. Further, we point out that Tabs. I and II include information gleaned from the previous sections to demonstrate that both polarization and directivity properties of the array may now be accounted for. We see that for all of the calculated cases, the number of elements required to maintain a $0.5 \%$ leakage are consistent with those which are required to keep $\sigma_{i}<<1$. Should cases be found where these are not the case, one should choose the higher number of elements.

\section{CONCLUSION}

For an array of dual-polarized orthogonal Hertzian dipoles, the polarimeter's Jones IXR at the intended beam scanning direction is unaffected by element rotation error; this is approximately true for practical dual-polarized (such as half-wavelength) dipoles for small rotation errors. When the rotation error is random and the number of elements very large, at the intended beam scanning, the relative Jones matrix estimation error converges to that of the error-free case, however, rotation error affects array directivity and radiation pattern. To quantify the relation between rotation error, crosspolarization levels of the elements and number of elements, we presented calculations involving an unpolarized source where the number of elements required to achieve a target crosspolarization correlation leakage may be estimated. Depending on the target, rotation error, and the Jones matrix of the antenna, the required "large" number may vary from tens to thousands of elements. Conversely, when the number of (large) elements are fixed, one may compute the rotation error required to maintain a cross-polarization correlation leakage. Our calculations for 289 elements in an SKA LFAA station show that a moderate antenna raw-cross polarization of 0.2 requires rotation standard deviation of approximately $6.1^{\circ}$.

\section{ACKNOWLEDGMENT}

We acknowledge the Wajarri Yamatji people as the traditional owners of the Murchison Radio Astronomy Observatory site. We thank the MWA project and personnel for their support in this work. Discussions with R. Wayth, E. de Lera Acedo, N. Razavi-Ghods, B. Fiorelli, and J. G. bij de Vaate on this topic are gratefully acknowledged.

\section{APPENDIX A}

This appendix discusses the small azimuthal undulations seen in practical dipoles and their impact on our formulation. Fig. 5 reports the IXR variation seen in orthogonal halfwavelength dipoles as a function of $\phi$ for a few $\theta$. The IXR undulations has a period of $90^{\circ}$ and therefore are not $\phi$ invariant (the undulations decrease with decreasing dipole lengths and in the limit of Hertzian dipoles are non-existent).

The rotation error model described by $(2,4)$ is a unitary operation as (4) is a unitary matrix and therefore does not reflect the rotation of the IXR pattern with rotation error. Thus, the applicability of $(2,4)$ is to be limited to small angles. For rotation errors of a few degrees, the expected IXR errors are small (of roughly $0.5 \mathrm{~dB}$ or less for $5^{\circ}$ error) as annotated in the figure below for the given $\theta$ angles. The impact of small IXR change to $1+2 / \sqrt{\text { IXR }}$, the first factor in the righthandside of (1), is also small. For instance for the annotated points $\left(\phi=20^{\circ}\right.$ and $\left.25^{\circ}\right)$ at $\theta=45^{\circ}$ in Fig. $5,1+2 / \sqrt{\mathrm{IXR}}=$ 1.41 and 1.39 , respectively, a difference of only $1.4 \%$.

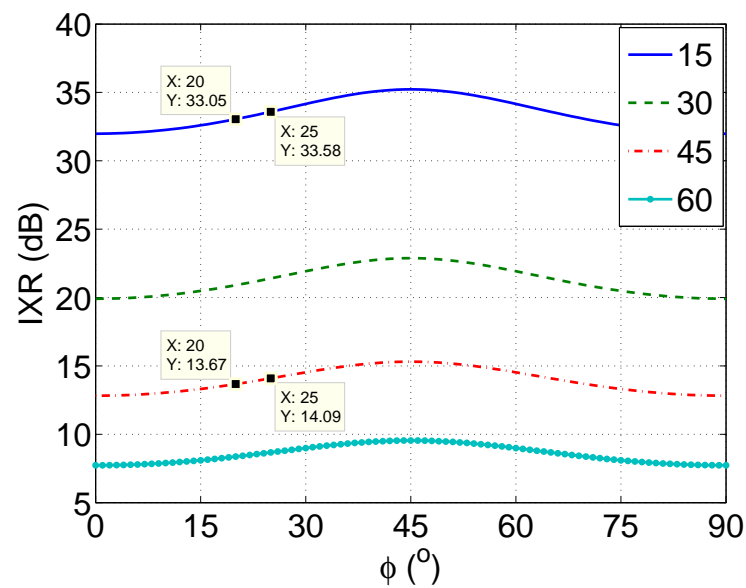

Figure 5. Azimuthal undulations calculated for orthogonal half-wavelength dipoles (assuming sinusoidal current distribution) at the indicated $\theta$ angles. Note that for small angles (such as $5^{\circ}$ ), the difference in IXR is approximately $0.5 \mathrm{~dB}$ or less.

\section{APPENDIX B}

This appendix is a commentary on Sec. III. We examine the possibility of a more general statement regarding IXR at $(\phi, \theta)$. From (10)

$$
\mathbf{F}=\left(\begin{array}{cc}
\mathbf{s}^{T} \mathbf{d}^{-} & 0 \\
0 & \mathbf{s}^{T}\left(\mathbf{d}^{-}\right)^{*}
\end{array}\right)
$$

In general, $\mathbf{s}^{T}\left(\mathbf{d}^{-}\right)^{*} \neq\left(\mathbf{s}^{T} \mathbf{d}^{-}\right)^{*}$ such that $\operatorname{IXR}\left(\mathbf{J}_{C}^{e r r}(\theta, \phi)\right) \neq$ $\operatorname{IXR}\left(\mathbf{J}_{C}(\theta, \phi)\right)$. However, if $\mathbf{s}$ is a real vector, which is fulfilled at $\left(\phi_{t}, \theta_{t}\right), \mathbf{s}^{T}\left(\mathbf{d}^{-}\right)^{*}=\left(\mathbf{s}^{T} \mathbf{d}^{-}\right)^{*}$ leading to (16). A full design study should involve IXR evaluation for the array in $(\theta, \phi)$ of interest which, in practice, is limited to the main beam. 
Next, we look at $\operatorname{IXR}(\phi, \theta)$ in an "average" sense. Let $\delta_{i}$ be a Gaussian random variable with zero mean and variance $\sigma^{2}$ (independent and identically distributed for $i=1 \ldots N$ ). The mean array factor is

$$
\begin{aligned}
E\left[F^{ \pm}\right] & =\frac{1}{N} E\left[\mathbf{s}^{T} \mathbf{d}^{ \pm}\right] \\
& =E\left[e^{ \pm j \delta_{i}}\right] F \\
& =e^{-\sigma^{2} / 2} F
\end{aligned}
$$

where $F=\mathbf{s}^{T}[1 \cdots 1] / N$ is the error-free array factor. The mean Jones matrix with error is given by

$$
E\left[\mathbf{J}_{C}^{e r r}\right]=e^{-\sigma^{2} / 2} F \mathbf{J}_{C}
$$

Thus if "average" IXR is computed as $\operatorname{IXR}\left(E\left[\mathbf{J}_{C}^{e r r}\right]\right)$, we obtain

$$
\operatorname{IXR}\left(E\left[\mathbf{J}_{C}^{e r r}(\theta, \phi)\right]\right)=\operatorname{IXR}\left(\mathbf{J}_{C}(\theta, \phi)\right)
$$

More rigorous investigation on this question requires treating the average IXR as $E\left[\operatorname{IXR}\left(\mathbf{J}_{C}^{e r r}\right)\right]$, which is beyond our current scope. However, the result in (34) leads us to conjecture, given the foregoing assumptions, that the average IXR is unaffected by random errors.

\section{REFERENCES}

[1] C. Lonsdale, et al., "The murchison widefield array: Design overview," Proceedings of the IEEE, vol. 97, no. 8, pp. 1497 -1506, aug. 2009.

[2] S. J. Tingay, et al., "The Murchison Widefield Array: The Square Kilometre Array Precursor at Low Radio Frequencies," Publications of the Astronomical Society of Australia, vol. 30, p. 7, Jan. 2013.

[3] S. J. Wijnholds and W. A. van Capellen, "In situ antenna performance evaluation of the LOFAR phased array radio telescope," IEEE Transactions on Antennas and Propagation, vol. 59, no. 6, pp. 1981 - 1989, June 2011.

[4] W. van Cappellen, S. Wijnholds, and J. Bregman, "Sparse antenna array configurations in large aperture synthesis radio telescopes," in Radar Conference, 2006. EuRAD 2006. 3rd European, sept. 2006, pp. 76 -79.

[5] S. W. Ellingson, "Sensitivity of antenna arrays for long-wavelength radio astronomy," IEEE Transactions on Antennas and Propagation, vol. 59, no. 6 , pp. 1855 - 1863, June 2011.

[6] (2010) SKA phase 1: Preliminary system decription. Online. SKA Project Development Office. Manchester, UK. [Online]. Available: http://www.skatelescope.org/publications/

[7] (2013) SKA1 system baseline design. Online. SKA Project Development Office. Manchester, UK. [Online]. Available: http://www.skatelescope.org/publications/request-for-proposals/

[8] A. Jiwani, S. Padhi, M. Waterson, P. Hall, and J. bij de Vaate, "Candidate wire spiral antennas for the ska radio telescope," in Electromagnetics in Advanced Applications (ICEAA), 2012 International Conference on, sept. 2012, pp. $510-513$.

[9] E. de Lera Acedo, "SKALA: A log-periodic antenna for the SKA," in Electromagnetics in Advanced Applications (ICEAA), 2012 International Conference on, sept. 2012, pp. $353-356$.

[10] M. Arts and B. Fiorelli, "Polarization studies of vivaldi aperture arrays for the square kilometre array," in Electromagnetics in Advanced Applications (ICEAA), 2013 International Conference on, 2013, pp. 356-359.

[11] P. Hall, et al., "First results from AAVS 0.5: A prototype array for next-generation radio astronomy," in Electromagnetics in Advanced Applications (ICEAA), 2013 International Conference on, 2013, pp. 340-343.

[12] T. D. Carozzi and G. Woan, "A fundamental figure of merit for radio polarimeters," IEEE Transactions on Antennas and Propagation, vol. 59, no. 6, pp. 2058-2065, June 2011.

[13] M. Ivashina, O. Iupikov, R. Maaskant, W. van Cappellen, and T. Oosterloo, "An optimal beamforming strategy for wide-field surveys with phased-array-fed reflector antennas," Antennas and Propagation, IEEE Transactions on, vol. 59, no. 6, pp. $1864-1875$, june 2011.
[14] A. Jiwani, T. Colegate, N. Razavi-Ghods, P. J. Hall, S. Padhi, and J. Geralt bij de Vaate, "Square Kilometre Array station configuration using two-stage beamforming," ArXiv e-prints, Nov. 2012.

[15] A. Sutinjo and P. Hall, "Element rotation tolerance in a low-frequency aperture array polarimeter," in Electromagnetics in Advanced Applications (ICEAA), 2013 International Conference on, 2013, pp. 58-61.

[16] T. D. Carozzi, G. Woan, and R. Maaskant, "Polarization Diversity for SKA Wide-field Polarimetry," in Wide Field Astronomy Technology for the Square Kilometre Array, 2009.

[17] A. Sutinjo and P. Hall, "Intrinsic cross-polarization ratio of dual-linearly polarized antennas for low-frequency radio astronomy," Antennas and Propagation, IEEE Transactions on, vol. 61, no. 5, pp. 2852-2856, 2013.

[18] H. Aumann and E. Anesta, "Polarization ratio improvement in a spiral element array," in Antennas and Propagation Society International Symposium, 2009. APSURSI '09. IEEE, june 2009, pp. 1 -4.

[19] G. B. Taylor, C. L. Carilli, and R. A. Perley, "Synthesis imaging in radio astronomy II," vol. 180, 1999. [Online]. Available: http://adsabs.harvard.edu/abs/1999ASPC..180.....T

[20] J. Ruze, "Antenna tolerance theory-a review," Proceedings of the IEEE, vol. 54, no. 4, pp. 633 - 640, april 1966.

[21] R. E. Collin and F. J. Zucker, Eds., Antenna Theory, Part. 1. McGrawHill, 1969, ch. 6.

[22] R. C. Hansen, Phased Array Antennas. Wiley-Interscience, 1998.

[23] R. J. Mailloux, Phased Array Antenna Handbook, 3rd ed. Artech House, 2005

[24] Y. Lo, "A mathematical theory of antenna arrays with randomly spaced elements," Antennas and Propagation, IEEE Transactions on, vol. 12, no. 3, pp. $257-268$, may 1964.

[25] K. F. Warnick, M. Ivashina, S. Wijnholds, and R. Maaskant, "Polarimetry with phased array antennas: Theoretical framework and definitions," Antennas and Propagation, IEEE Transactions on, vol. 60, no. 1, pp. 184-196, 2012.

[26] O. M. Smirnov, "Revisiting the radio interferometer measurement equation. I. A full-sky Jones formalism," Astron.Astrophys., vol. 527, p. A106, 2011. 